\title{
On Soft Ordered Maps
}

\author{
T. M. Al-shami ${ }^{\text {} l}$, M. E. El-Shafei ${ }^{2}$ and M. Abo-Elhamayel ${ }^{3}$ \\ 1,2,3 Department of Mathematics, Mansoura University, Mansoura, Egypt \\ ${ }^{1}$ Department of Mathematics, Sana'a University, Sana'a, Yemen \\ ${ }^{1}$ tareqalshami83@gmail.com, ${ }^{2}$ meshafei@hotmail.com, ${ }^{3}$ mohamedaboelhamayel@yahoo.com
}

\begin{abstract}
In the current work, we define soft $\lambda$-continuous, soft $\lambda$-open, soft $\lambda$-closed and soft $\lambda$-homeomorphism maps via soft topological ordered spaces, where $\lambda \in\{I, D, B\}$. The relationships among these soft maps are shown with the help of examples and their main properties are studied. In this regard, the equivalent conditions for each one of these soft maps are investigated, and an enough condition for the equivalent between soft $\lambda$-open and soft $\lambda$-closed maps is given for each $\lambda$. Also, we discuss the interrelations between these soft maps and their counterparts on topological ordered spaces and clarify a significant role of extended soft topologies in this point. In the end, we point out under what conditions the initiated soft maps preserve some soft ordered separation axioms, and conclude the behaviors of these soft maps under some compositions.
\end{abstract}

Keywords: Soft $I(D, B)$-continuous map, soft $I(D, B)$-open map, soft $I(D, B)$-homeomorphism map, soft ordered separation axioms

2010 MSC No: 54F05, 54F15

\section{Introduction and Preliminaries}

The concept of topological ordered spaces was first introduced and systematically studied by Nachbin 33 . He investigated the main properties of increasing and decreasing sets and verified some interesting results associated with the normally ordered spaces. Then McCartan [31, in 1968, carried out a detailed study on ordered separation axioms. He focused on characterizing $T_{i}$-ordered spaces $(i=0,1,2)$, regularly ordered and normality ordered spaces. The concepts of continuity, openness, closedness and homeomorphism between topological ordered spaces were presented and studied by Kumar [24. For more investigation on topological ordered spaces, interested reader can find that from [11, 22, 25, 26, 27]. Das [14], in 2004, generalized ordered topologies to ordered supra topologies. Then many studies on supra topological ordered spaces were done (see, for example, [3, 5, 7, 8, 16, 17, 18]).

During recent past, the researchers endeavoured to approach uncertainty with some tools other than probability theory and fuzzy set theory. Soft set theory, was initiated by Molotdsov [32, has an important contribution towards the provision of such a mathematical tool to resolve the issues related to ambiguity and uncertainty in data based problems arising in mathematics or related disciplines. The ease of the definition of soft set made it more active to apply in many situations arising in information sciences, decision making, forecasting, demand analysis, mathematics and many other related disciplines as well. We refer reader for more studies on soft sets to ([1, 2, 4, 20, 28, 29, 30, ).

Shabir and Naz [37] employed the notion of soft sets to initiate the concept of soft topological spaces. They formulated and studied the basic notions on soft topological spaces such as soft interior points of a soft set, soft subspaces and soft separation axioms. Later on, numerous studies were done on soft topological notions and their

\footnotetext{
${ }^{*}$ Corresponding author. T. M. Al-shami ${ }^{1}$ tareqalshami83@gmail.com
} 
features (see, for example, [6, 10, 12, 15, 23, 34, 35, 36]). Recently, El-Shafei et al. [19] established two new soft relations, namely partial belong and total non belong. Depend on them, they introduced partial soft separation axioms, namely p-soft $T_{i}$-spaces for $i=0,1,2,3,4$. With regard to our topic, Al-shami et al. [9] initiated a concept of soft topological ordered spaces. They gave the fundamental properties of a soft partially ordered sets and then defined p-soft $T_{i}$-ordered spaces for $i=0,1,2,3,4$.

Since soft continuity, soft openness, soft closedness and soft homeomorphism are very important concepts in soft topology, it is natural to think about its soft order topology analogue. So the aim here, is to introduce and study new types of soft maps via soft topological ordered spaces, namely soft $\lambda$-continuous, soft $\lambda$-open, soft $\lambda$-closed and soft $\lambda$-homeomorphism maps, where $\lambda \in\{I, D, B\}$. The purpose of establishing various examples is twofold, one is to show the relationships among these soft maps introduced herein and the other is to illustrate that soft $\lambda$-continuous, soft $\lambda$-open, soft $\lambda$-closed and soft $\lambda$-homeomorphism maps are strictly stronger than soft continuous, soft open, soft closed and soft homeomorphism maps, respectively, for each $\lambda$. Also, we completely describe each one of the initiated soft maps and clarify a significant role of extended soft topologies on studying the interrelations between these soft maps and their counterparts on topological ordered spaces. Ultimately, we point out the interrelations between these soft maps and some soft ordered separation axioms and probe the behaviors of these maps under some compositions.

The following portion of this section is devoted to recalling some definitions and results that will be needed in the sequel.

Definition 1.1. [32] An ordered pair $(G, E)$ is said to be a soft set over $X$ if $G$ is a map of a set of parameters $E$ into $2^{X}$.

For short, we use the notation $G_{E}$ instead of $(G, E)$ and we write it as $G_{E}=\left\{(e, G(e)): e \in E\right.$ and $\left.G(e) \in 2^{X}\right\}$.

Definition 1.2. [30] A soft set $G_{E}$ over $X$ is called a null soft set, denoting by $\widetilde{\emptyset}$, if $G(e)=\emptyset$ for each e $\in E$; and it is called an absolute soft set, denoting by $\tilde{X}$, if $G(e)=X$ for each $e \in E$.

Definition 1.3. [4] The relative complement of a soft set $G_{E}$ is denoted by $G_{E}^{c}$, where $G^{c}: E \rightarrow 2^{X}$ is a mapping defined by $G^{c}(e)=X \backslash G(e)$ for each $e \in E$.

In this connection, it is worth noting that $x \notin G_{E}$ does not imply that $x \in G_{E}^{c}$.

Definition 1.4. [4] $A$ soft set $G_{A}$ is a soft subset of a soft set $F_{B}$, denoted by $G_{A} \subseteq F_{B}$, if $A \subseteq B$ and $G(a) \subseteq F(a)$ for all $a \in A$.

The soft sets $G_{A}$ and $F_{B}$ are soft equal if each of them is a soft subset of the other.

Definition 1.5. 37 For $x \in X$ and a soft set $G_{E}$ over $X$, we say that $x \in G_{E}$ if $x \in G(e)$ for each $e \in E$.

Definition 1.6. [37] A collection $\tau$ of soft sets over $X$ under a parameters set $E$ is said to be a soft topology on $X$ if the following three conditions hold:

(i) $\widetilde{X}$ and $\widetilde{\emptyset}$ belong to $\tau$.

(ii) The soft intersection of finite members in $\tau$ belongs to $\tau$.

(iii) The soft union of any members in $\tau$ belongs to $\tau$.

The triple $(X, \tau, E)$ is called a soft topological space. Every member of $\tau$ is called soft open and its relative complement is called soft closed. The soft closure of a soft set $H_{E}\left(\right.$ briefly cl $\left.\left(H_{E}\right)\right)$ is the smallest soft closed set that contains $H_{E}$; and the soft interior of a soft set $H_{E}$ (briefly int $\left(H_{E}\right)$ ) is the largest soft open set that is contained in $H_{E}$. $A$ collection of all soft subsets of the absolute soft set $\widetilde{X}$ is denoted by $S\left(X_{E}\right)$.

Proposition 1. 37] Let $(X, \tau, E)$ be a soft topological space. Then $\tau_{e}=\left\{G(e): G_{E} \in \tau\right\}$ defines a topology on $X$ for each $e \in E$.

Proposition 2. 34 Consider $(X, \tau, E)$ is a soft topological space and $\tau_{e}$ is a topology on $X$ as in the above proposition. Then $\tau^{\star}=\left\{G_{E}: G(e) \in \tau_{e}\right.$ for each $\left.e \in E\right\}$ is a soft topology on $X$ finer than $\tau$.

Remark 1.1. Henceforth, we called $\tau_{e}$ and $\tau^{\star}$, which given in the two proposition above, a parametric topology and an extended soft topology, respectively.

We modify the definition of a soft map given in [38] to be as follows. 
Definition 1.7. Consider $f: X \rightarrow Y$ and $\phi: A \rightarrow B$ are two maps and let $f_{\phi}: S\left(X_{A}\right) \rightarrow S\left(Y_{B}\right)$ be a soft map. Let $G_{K}$ and $H_{L}$ be soft subsets of $S\left(X_{A}\right)$ and $S\left(Y_{B}\right)$, respectively. The image of $G_{K}$ and pre-image of $H_{L}$ are defined as follows:

(i) $f_{\phi}\left(G_{K}\right)=\left(f_{\phi}(G)\right)_{B}$ is a soft subset of $S\left(Y_{B}\right)$ such that

$$
f_{\phi}(G)(b)=\left\{\begin{array}{ccc}
\bigcup_{a \in \phi^{-1}(b) \cap K} f(G(a)) & : & \phi^{-1}(b) \bigcap K \neq \emptyset \\
\emptyset & : & \phi^{-1}(b) \bigcap K=\emptyset
\end{array}\right.
$$

for each $b \in B$.

(ii) $f_{\phi}^{-1}\left(H_{L}\right)=\left(f_{\phi}^{-1}(H)\right)_{A}$ is a soft subset of $S\left(X_{A}\right)$ such that

$$
f_{\phi}^{-1}(H)(a)=\left\{\begin{array}{ccc}
f^{-1}(H(\phi(a))) & : & \phi(a) \in L \\
\emptyset & : & \phi(a) \notin L
\end{array}\right.
$$

for each $a \in A$.

Remark 1.2. Henceforward, a soft map $f_{\phi}: S\left(X_{A}\right) \rightarrow S\left(Y_{B}\right)$ means that we have a map $f$ of the universe set $X$ into the universe set $Y$ and a map $\phi$ of the set of parameters $A$ into the set of parameters $B$

Definition 1.8. [38] A soft map $f_{\phi}: S\left(X_{A}\right) \rightarrow S\left(Y_{B}\right)$ is said to be injective (resp. surjective, bijective) if $f$ and $\phi$ are injective (resp. surjective, bijective).

Proposition 3. 34 Consider $f_{\phi}: S\left(X_{A}\right) \rightarrow S\left(Y_{B}\right)$ is a soft map and let $G_{A}$ and $H_{B}$ be two soft subsets of $S\left(X_{A}\right)$ and $S\left(Y_{B}\right)$, respectively. Then we have the following results:

(i) $G_{A} \widetilde{\subseteq} f_{\phi}^{-1} f_{\phi}\left(G_{A}\right)$ and the equality relation holds if $f_{\phi}$ is injective.

(ii) $f_{\phi} f_{\phi}^{-1}\left(H_{B}\right) \widetilde{\widetilde{C}} H_{B}$ and the equality relation holds if $f_{\phi}$ is surjective.

Definition 1.9. ([34], [38]) A soft map $f_{\phi}:(X, \tau, A) \rightarrow(Y, \theta, B)$ is said to be:

(i) Soft continuous if the inverse image of each soft open subset of $(Y, \theta, B)$ is a soft open subset of $(X, \tau, A)$.

(ii) Soft open (resp. soft closed) if the image of each soft open (resp. soft closed) subset of $(X, \tau, A)$ is a soft open (resp. soft closed) subset of $(Y, \theta, B)$.

(iii) Soft homeomorphism if it is bijective, soft continuous and soft open.

Definition 1.10. ([15], [34]) $A$ soft set $P_{E}$ over $X$ is called soft point if there exists $e \in E$ and there exists $x \in X$ such that $P(e)=\{x\}$ and $P(\alpha)=\emptyset$ for each $\alpha \in E \backslash\{e\}$. A soft point will be shortly denoted by $P_{e}^{x}$ and we say that $P_{e}^{x} \in G_{E}$, if $x \in G(e)$.

Definition 1.11. [19] For $x \in X$ and a soft set $G_{E}$ over $X$, we say that $x \Subset G_{E}$ if $x \in G(e)$ for some $e \in E$.

Proposition 4. [19] Consider $x \in X, y \in Y, G_{A} \in S\left(X_{A}\right)$ and $H_{B} \in S\left(Y_{B}\right)$ and let $f_{\phi}: S\left(X_{A}\right) \rightarrow S\left(Y_{B}\right)$ be a soft map. Then we have the following results:

(i) If $\phi$ is surjective and $x \in G_{A}$, then $f(x) \in f_{\phi}\left(G_{A}\right)$.

(ii) If $y \in H_{B}$, then $x \in f_{\phi}^{-1}\left(H_{B}\right)$ for each $x \in f^{-1}(y)$.

(iii) If $f$ is injective and $x \notin G_{A}$, then $f(x) \notin f_{\phi}\left(G_{A}\right)$.

(iv) If $y \notin H_{B}$, then $x \notin f_{\phi}^{-1}\left(H_{B}\right)$ for each $x \in f^{-1}(y)$.

Definition 1.12. [19] A soft topological space $(X, \tau, E)$ is called:

(i) $p$-soft $T_{0}$ if for every pair of distinct points $x, y \in X$, there is a soft open set $G_{E}$ such that $x \in G_{E}, y \notin G_{E}$ or $y \in G_{E}, x \notin G_{E}$.

(ii) $p$-soft $T_{1}$ if for every pair of distinct points $x, y \in X$, there are soft open sets $G_{E}$ and $F_{E}$ such that $x \in G_{E}, y \notin$ $G_{E}$ and $y \in F_{E}, x \notin F_{E}$. 
(iii) $p$-soft $T_{2}$ if for every pair of distinct points $x, y \in X$, there are disjoint soft open sets $G_{E}$ and $F_{E}$ containing $x$ and $y$, respectively.

(iv) $p$-soft regular if for every soft closed set $H_{E}$ and $x \in X$ such that $x \notin H_{E}$, there are disjoint soft open sets $G_{E}$ and $F_{E}$ such that $H_{E} \subseteq G_{E}$ and $x \in F_{E}$.

(vi) 37 Soft normal if for every two disjoint soft closed sets $H_{1_{E}}$ and $H_{2_{E}}$, there are two disjoint soft open sets $G_{E}$ and $F_{E}$ such that $H_{1_{E}} \widetilde{\simeq} G_{E}$ and $H_{2_{E}} \widetilde{\widetilde{C}} F_{E}$.

(vii) p-soft $T_{3}$ (resp. p-soft $T_{4}$ ) if it is both p-soft regular (resp. soft normal) and p-soft $T_{1}$.

Definition 1.13. [9] Let $\preceq$ be a partial order relation on a non-empty set $X$ and let $E$ be a set of parameters. $A$ triple $(X, E, \preceq)$ is said to be a partially ordered soft set.

Proposition 5. [19] The following two results hold for a soft map $f_{\phi}: S\left(X_{A}\right) \rightarrow S\left(Y_{B}\right)$ :

(i) The image of each soft point is soft point.

(ii) The inverse image of each soft point is soft point provided that $f$ is bijective.

Definition 1.14. [9] We define an increasing soft operator $i:\left(S\left(X_{E}\right), \preceq\right) \rightarrow\left(S\left(X_{E}\right), \preceq\right)$ and a decreasing soft operator $d:\left(S\left(X_{E}\right), \preceq\right) \rightarrow\left(S\left(X_{E}\right), \preceq\right)$ for each soft subset $G_{E}$ of $S\left(X_{E}\right)$ as follows:

(i) $i\left(G_{E}\right)=(i G)_{E}$, where $i G$ is a mapping of $E$ into $X$ given by $i G(e)=i(G(e))=\{x \in X: y \preceq x$ for some $y \in G(e)\}$.

(ii) $d\left(G_{E}\right)=(d G)_{E}$, where $d G$ is a mapping of $E$ into $X$ given by $d G(e)=d(G(e))=\{x \in X: x \preceq y$ for some $y \in G(e)\}$.

Definition 1.15. [9] A soft subset $G_{E}$ of a partially ordered soft set $(X, E, \preceq)$ is said to be increasing (resp. decreasing) if $G_{E}=i\left(G_{E}\right)\left(\right.$ resp. $\left.G_{E}=d\left(G_{E}\right)\right)$.

Theorem 1.1. [9] If a soft map $f_{\phi}:\left(S\left(X_{A}\right), \preceq_{1}\right) \rightarrow\left(S\left(Y_{B}\right), \preceq_{2}\right)$ is increasing, then the inverse image of each increasing (resp. decreasing) soft subset of $\widetilde{Y}$ is an increasing (resp. a decreasing) soft subset of $\widetilde{X}$.

Definition 1.16. [9] A quadrable system $(X, \tau, E, \preceq)$ is said to be a soft topological ordered space, where $(X, \tau, E)$ is a soft topological space and $(X, E, \preceq)$ is a partially ordered soft set. Through this work, we use the two notations $\left(X, \tau, E, \preceq_{1}\right)$ and $\left(Y, \theta, F, \preceq_{2}\right)$ to refer to soft topological ordered spaces.

Definition 1.17. [9] A soft topological ordered space $(X, \tau, E, \preceq)$ is said to be:

(i) Lower (resp. Upper) p-soft $T_{1}$-ordered if for every $x \npreceq y$ in $X$, there exists an increasing (resp. a decreasing) soft neighborhood $W_{E}$ of $x$ (resp. $y$ ) such that $y \notin W_{E}$ (resp. $x \notin W_{E}$ ).

(ii) $p$-soft $T_{0}$-ordered if it is lower soft $T_{1}$-ordered or upper soft $T_{1}$-ordered.

(iii) $p$-soft $T_{1}$-ordered if it is lower soft $T_{1}$-ordered and upper soft $T_{1}$-ordered.

(iv) $p$-soft $T_{2}$-ordered if for every $x \npreceq y$ in $X$, there exist disjoint soft neighborhoods $W_{E}$ and $V_{E}$ of $x$ and $y$, respectively, such that $W_{E}$ is increasing and $V_{E}$ is decreasing.

(v) Lower (resp. Upper) p-soft regularly ordered if for each decreasing (resp. increasing) soft closed set $H_{E}$ and $x \in X$ such that $x \notin H_{E}$, there exist disjoint soft neighbourhoods $W_{E}$ of $H_{E}$ and $V_{E}$ of $x$ such that $W_{E}$ is decreasing (resp. increasing) and $V_{E}$ is increasing (resp. decreasing).

(vi) -soft regularly ordered if it is both lower p-soft regularly ordered and upper p-soft regularly ordered.

(vii) Lower (resp. Upper) p-soft $T_{3}$-ordered if it is both lower (resp. upper) p-soft $T_{1}$-ordered and lower (resp. upper) p-soft regularly ordered.

(viii) $p$-soft $T_{3}$-ordered if it is both lower $p$-soft $T_{3}$-ordered and upper $p$-soft $T_{3}$-ordered.

(ix) Soft normally ordered if for each disjoint soft closed sets $F_{E}$ and $H_{E}$ such that $F_{E}$ is increasing and $H_{E}$ is decreasing, there exist disjoint soft neighbourhoods $W_{E}$ of $F_{E}$ and $V_{E}$ of $H_{E}$ such that $W_{E}$ is increasing and $V_{E}$ is decreasing. 
(x) $p$-soft $T_{4}$-ordered if it is soft normally ordered and $p$-soft $T_{1}$-ordered.

Definition 1.18. 24 $A$ map $f:\left(X, \tau, \preceq_{1}\right) \rightarrow\left(Y, \theta, \preceq_{2}\right)$ is said to be:

(i) I (resp. D, B) -continuous if the inverse image of each open set is I (resp. D, B) -open.

(ii) $I$ (resp. D, B) -open if the image of each open set is I (resp. D, B) -open.

(iii) I (resp. D, B) -closed if the image of each open set is I (resp. D, B)-closed.

(iv) I (resp. D, B) -homeomorphism if it is bijective, I (resp. D, B) -continuous and I (resp. D, B) -open.

Throughout this manuscript $\lambda \in\{I, D, B\}$.

\section{Soft $\lambda$-continuous maps}

In this section, we give some kinds of soft continuity via soft topological ordered spaces and illustrate the relationships among them with the help of examples. We then characterize each one of the introduced soft maps and study the pre image of p-soft $T_{i}$-spaces $(i=0,1,2)$ under these types of soft continuous maps.

Definition 2.1. A soft subset $H_{E}$ of $\left(X, \tau, E, \preceq_{1}\right)$ is said to be balancing if it is increasing and decreasing.

Definition 2.2. A soft subset $H_{E}$ of $\left(X, \tau, E, \preceq_{1}\right)$ is said to be:

(i) Soft I (resp. Soft D, Soft B) -open if it is soft open and increasing (resp. decreasing, balancing).

(ii) Soft I (resp. Soft D, Soft B) -closed if it is soft closed and increasing (resp. decreasing, balancing).

Definition 2.3. A soft map $f_{\phi}:\left(X, \tau, E, \preceq_{1}\right) \rightarrow\left(Y, \theta, F, \preceq_{2}\right)$ is said to be:

(i) Soft I (resp. Soft D, Soft B) -continuous at $P_{e}^{x} \in \widetilde{X}$ if for each soft open set $H_{F}$ containing $f_{\phi}\left(P_{e}^{x}\right)$, there exists a soft $I$ (resp. soft $D$, soft $B$ ) -open set $G_{E}$ containing $P_{e}^{x}$ such that $f_{\phi}\left(G_{E}\right) \widetilde{\subseteq} H_{F}$.

(ii) Soft I (resp. Soft D, Soft B) -continuous at $x \in X$ if it is soft I (resp. soft D, soft B) -continuous at each $P_{e}^{x}$.

(iii) Soft I (resp. Soft D, Soft B) -continuous if it is soft I (resp. soft D, soft B) -continuous at each $x \in X$.

Theorem 2.1. A soft map $f_{\phi}:\left(X, \tau, E, \preceq_{1}\right) \rightarrow\left(Y, \theta, F, \preceq_{2}\right)$ is soft I (resp. soft D, soft B) -continuous if and only if the inverse image of each soft open subset of $\widetilde{Y}$ is a soft I (resp. soft D, soft B) -open subset of $\widetilde{X}$.

Proof. We only present the proof in the case of $f_{\phi}$ is soft $I$-continuous and the cases between parenthesis can be made similarly.

To prove the necessary part, let $G_{F}$ be a soft open subset of $\widetilde{Y}$. Since the proof is trivial if $f_{\phi}^{-1}\left(G_{F}\right)=\widetilde{\emptyset}$, then we consider $f^{-1}\left(G_{F}\right) \neq \widetilde{\emptyset}$. By choosing $P_{e}^{x} \in \widetilde{X}$ such that $P_{e}^{x} \in f_{\phi}^{-1}\left(G_{F}\right)$, we obtain $f_{\phi}\left(P_{e}^{x}\right) \in G_{F}$. So there exists a soft $I$-open set $H_{E}$ containing $P_{e}^{x}$ such that $f_{\phi}\left(H_{E}\right) \widetilde{\subseteq} G_{F}$. Since $P_{e}^{x}$ is chosen arbitrary, then $f_{\phi}^{-1}\left(G_{F}\right)=\widetilde{U}_{P_{e}^{x} \in f_{\phi}^{-1}\left(G_{F}\right)} H_{E}$. Thus $f_{\phi}^{-1}\left(G_{F}\right)$ is a soft $I$-open subset of $\tilde{X}$.

To prove the sufficient part, let $G_{F}$ be a soft open subset of $\tilde{Y}$ containing $f_{\phi}\left(P_{e}^{x}\right)$. Then $P_{e}^{x} \in f_{\phi}^{-1}\left(G_{F}\right)$. By hypothesis, $f_{\phi}^{-1}\left(G_{F}\right)$ is a soft $I$-open set. Since $f_{\phi}\left(f_{\phi}^{-1}\left(G_{F}\right)\right) \widetilde{\widetilde{C}} G_{F}$, then $f_{\phi}$ is a soft $I$-continuous at $P_{e}^{x} \in X$ and since $P_{e}^{x}$ is chosen arbitrary, then $f_{\phi}$ is soft $I$-continuous.

Remark 2.1. From Definition 2.3 , we can note the following:

(i) Every soft I (soft D, soft B) -continuous map is always soft continuous.

(ii) Every soft B-continuous map is soft I-continuous (soft D-continuous).

To elucidate that a soft $B$-continuous map is real generalization of soft $I$-continuous and soft $D$-continuous maps, we construct the following two examples. 
Example 2.1. Let the two universe sets $X=\{h, i, j\}, Y=\{w, z\}$ and the two parameters sets $A=\left\{a_{1}, a_{2}\right\}$, $B=\left\{b_{1}, b_{2}\right\}$. Consider a map $\phi: A \rightarrow B$ is defined as, $\phi\left(a_{m}\right)=b_{m}$ for $m \in\{1,2\}$ and a map $f: X \rightarrow Y$ is defined as, $f(h)=w$ and $f(i)=f(j)=z$. We define a partial order relation on $X$ as $\preceq=\triangle \bigcup\{(i, h),(h, j),(i, j)\}$ and we define two soft topologies $\tau$ and $\theta$ on $X$ and $Y$, respectively, as $\tau=\left\{\widetilde{\emptyset}, \widetilde{X}, F_{A}, G_{A}\right\}$ and $\theta=\left\{\widetilde{\emptyset}, \widetilde{Y}, H_{B}\right\}$, where $F_{A}=\left\{\left(a_{1}, X\right),\left(a_{2},\{h\}\right)\right\}, G_{A}=\left\{\left(a_{1},\{h, i\}\right),\left(a_{2},\{h\}\right)\right\}$ and $H_{B}=\left\{\left(b_{1}, Y\right),\left(b_{2},\{w\}\right)\right\}$. Then one can readily check that a soft map $f_{\phi}: S\left(X_{A}\right) \rightarrow S\left(Y_{B}\right)$ is soft continuous. On the other hand, $f_{\phi}^{-1}\left(H_{B}\right)=F_{A}$ is neither a soft D-open nor a soft I-open set. Hence $f_{\phi}$ is not soft I (soft D, soft B) -continuous.

Example 2.2. In Example above, if we replace only the partial order relation by $\preceq=\triangle \bigcup\{(h, j),(i, j)\}($ resp. $\preceq=$ $\triangle \bigcup\{(i, h)\})$, then the soft map $f_{\phi}$ is soft D-continuous (resp. soft I-continuous), but is not soft B-continuous.

Definition 2.4. For a soft subset $H_{E}$ of $(X, \tau, E, \preceq)$, we define the following six operators:

(i) $H_{E}^{i o}\left(r e s p . H_{E}^{d o}, H_{E}^{b o}\right)$ is the largest soft I (resp. soft D, soft B) -open set that is contained in $H_{E}$.

(ii) $H_{E}^{i c l}\left(\right.$ resp. $\left.H_{E}^{d c l}, H_{E}^{b c l}\right)$ is the smallest soft I (resp. soft D, soft B)-closed set that contains $H_{E}$.

Lemma 2.1. We have the following three properties for a soft subset $H_{E}$ of $(X, \tau, E, \preceq)$ :

(i) $\left(H_{E}^{d c l}\right)^{c}=\left(H_{E}^{c}\right)^{i o}$.

(ii) $\left(H_{E}^{i c l}\right)^{c}=\left(H_{E}^{c}\right)^{d o}$.

(iii) $\left(H_{E}^{b c l}\right)^{c}=\left(H_{E}^{c}\right)^{b o}$.

Proof. (i) $\left(H_{E}^{d c l}\right)^{c}=\left\{\widetilde{U} F_{E}: F_{E} \text { is a soft } D \text {-closed set containing } H_{E}\right\}^{c}$

$=\widetilde{\bigcap}\left\{F_{E}^{c}: F_{E}^{c}\right.$ is a soft $I$-open set contained in $\left.H_{E}^{c}\right\}=\left(H_{E}^{c}\right)^{i o}$.

By analogy with (i), one can prove (ii) and (iii).

Theorem 2.2. The following five properties of a soft map $f_{\phi}:\left(X, \tau, E \preceq_{1}\right) \rightarrow\left(Y, \theta, F, \preceq_{2}\right)$ are equivalent:

(i) $f_{\phi}$ is soft I-continuous;

(ii) $f_{\phi}^{-1}\left(L_{F}\right)$ is a soft $D$-closed subset of $\widetilde{X}$ for each soft closed subset $L_{F}$ of $\tilde{Y}$;

(iii) $\left(f_{\phi}^{-1}\left(M_{F}\right)\right)^{d c l} \widetilde{\subseteq} f_{\phi}^{-1}\left(\operatorname{cl}\left(M_{F}\right)\right)$ for every $M_{F} \widetilde{\simeq} \widetilde{Y}$;

(iv) $f_{\phi}\left(N_{E}^{d c l}\right) \widetilde{\subseteq} c l\left(f_{\phi}\left(N_{E}\right)\right)$ for every $N_{E} \widetilde{\subseteq} \widetilde{X}$;

(v) $f_{\phi}^{-1}\left(\operatorname{int}\left(M_{F}\right)\right) \widetilde{\subseteq}\left(f_{\phi}^{-1}\left(M_{F}\right)\right)^{\text {io }}$ for every $M_{F} \widetilde{\subseteq} \widetilde{Y}$.

Proof. (i) $\Rightarrow$ (ii) : Consider $L_{F}$ is a soft closed subset of $\tilde{Y}$. By hypothesis, $f_{\phi}^{-1}\left(L_{F}^{c}\right)$ is a soft $I$-open subset of $\widetilde{X}$ and by the fact that $f_{\phi}^{-1}\left(L_{F}^{c}\right)=\left(f_{\phi}^{-1}\left(L_{F}\right)\right)^{c}$, we obtain $f_{\phi}^{-1}\left(L_{F}\right)$ is soft $D$-closed as required.

(ii) $\Rightarrow$ (iii) : It follows from statement (ii) that $f_{\phi}^{-1}\left(\operatorname{cl}\left(M_{E}\right)\right)$ is a soft $D$-closed subset of $\tilde{X}$ for every $M_{F} \widetilde{\subseteq} \tilde{Y}$. So $\left(f_{\phi}^{-1}\left(M_{F}\right)\right)^{d c l} \widetilde{\simeq}\left(f_{\phi}^{-1}\left(c l\left(M_{F}\right)\right)^{d c l}=f_{\phi}^{-1}\left(c l\left(M_{F}\right)\right)\right.$.

(iii) $\Rightarrow$ (iv) : From the fact that $N_{E}^{d c l} \widetilde{\subseteq}\left(f_{\phi}^{-1}\left(f_{\phi}\left(N_{E}\right)\right)^{d c l}\right.$ and from (iii), we have $\left(f_{\phi}^{-1}\left(f_{\phi}\left(N_{E}\right)\right)^{d c l} \widetilde{\simeq} f_{\phi}^{-1}\left(c l\left(f_{\phi}\left(N_{E}\right)\right)\right)\right.$. This implies that $f_{\phi}\left(N_{E}^{d c l}\right) \widetilde{\widetilde{C}} c l\left(f_{\phi}\left(N_{E}\right)\right)$.

(iv) $\Rightarrow(\mathbf{v})$ : For any soft subset $M_{F}$ of $\widetilde{Y}$, we obtain from Lemma 2.1 that $f_{\phi}\left(\widetilde{X}-\left(f_{\phi}^{-1}\left(N_{E}\right)\right)^{i o}\right)=f_{\phi}\left(\left(\left(f_{\phi}^{-1}\left(N_{E}\right)\right)^{c}\right)^{d c l}\right)$. It follows from statement (iv), that $f_{\phi}\left(\left(\left(f_{\phi}^{-1}\left(N_{E}\right)\right)^{c}\right)^{d c l}\right) \widetilde{\widetilde{c}} c l\left(f_{\phi}\left(f_{\phi}^{-1}\left(N_{E}\right)\right)^{c}\right)=\operatorname{cl}\left(f_{\phi}\left(f_{\phi}^{-1}\left(N_{E}^{c}\right)\right)\right) \widetilde{\subseteq} c l\left(\widetilde{Y}-N_{E}\right)=\widetilde{Y}-$ $\operatorname{int}\left(N_{E}\right)$. Therefore $\left(\widetilde{X}-\left(f_{\phi}^{-1}\left(N_{E}\right)\right)^{i o}\right) \widetilde{\subseteq} f_{\phi}^{-1}\left(\widetilde{Y}-i n t\left(N_{E}\right)\right)=\widetilde{X}-f_{\phi}^{-1}\left(\operatorname{int}\left(N_{E}\right)\right)$. Thus $f_{\phi}^{-1}\left(\operatorname{int}\left(N_{E}\right)\right) \widetilde{\widetilde{C}}\left(f_{\phi}^{-1}\left(N_{E}\right)\right)^{i o}$. $(\mathbf{v}) \Rightarrow(\mathbf{i})$ : Consider $M_{F}$ is a soft open subset of $\tilde{Y}$. Then $f_{\phi}^{-1}\left(M_{F}\right)=f_{\phi}^{-1}\left(\operatorname{int}\left(M_{F}\right)\right) \widetilde{\subseteq}\left(f_{\phi}^{-1}\left(M_{F}\right)\right)^{i o}$. So $\left(f_{\phi}^{-1}\left(M_{F}\right)\right)^{i o}=f_{\phi}^{-1}\left(M_{F}\right)$ and this means that $f_{\phi}^{-1}\left(M_{F}\right)$ is a soft $I$-open subset of $\tilde{X}$. Hence the desired result is proved.

Theorem 2.3. The following five properties of a soft map $f_{\phi}:\left(X, \tau, E \preceq_{1}\right) \rightarrow\left(Y, \theta, F, \preceq_{2}\right)$ are equivalent:

(i) $f_{\phi}$ is soft D-continuous (resp. soft B-continuous);

(ii) $f_{\phi}^{-1}\left(L_{F}\right)$ is a soft I-closed (resp. soft B-closed) subset of $\tilde{X}$ for each soft closed subset $L_{F}$ of $\tilde{Y}$; 
(iii) $\left(f_{\phi}^{-1}\left(M_{F}\right)\right)^{i c l} \widetilde{\subseteq} f_{\phi}^{-1}\left(c l\left(M_{F}\right)\right)\left(\right.$ resp. $\left(f_{\phi}^{-1}\left(M_{F}\right)\right)^{b c l} \widetilde{\subseteq} f_{\phi}^{-1}\left(c l\left(M_{F}\right)\right)$ for every $M_{F} \widetilde{\subseteq} \widetilde{Y}$;

(iv) $f_{\phi}\left(N_{E}^{i c l}\right) \widetilde{\widetilde{\subseteq}} c l\left(f_{\phi}\left(N_{E}\right)\right)\left(\right.$ resp. $f_{\phi}\left(N_{E}^{b c l}\right) \widetilde{\widetilde{\subseteq}} c l\left(f_{\phi}\left(N_{E}\right)\right)$ for every $N_{E} \widetilde{\subseteq} \widetilde{X}$;

(v) $f_{\phi}^{-1}\left(\operatorname{int}\left(M_{F}\right)\right) \widetilde{\subseteq}\left(f_{\phi}^{-1}\left(M_{F}\right)\right)^{d o}\left(\right.$ resp. $f_{\phi}^{-1}\left(\operatorname{int}\left(M_{F}\right)\right) \widetilde{\subseteq}\left(f_{\phi}^{-1}\left(M_{F}\right)\right)^{\text {bo }}$ for every $M_{F} \widetilde{\widetilde{\subseteq}} \widetilde{Y}$.

Proof. The proof is similar to that of Theorem 2.2.

Theorem 2.4. If a soft map $g_{\phi}:\left(X, \tau, E, \preceq_{1}\right) \rightarrow\left(Y, \theta, F, \preceq_{2}\right)$ is soft I (resp. soft $D$, soft B) -continuous, then a map $g:\left(X, \tau_{e}, \preceq_{1}\right) \rightarrow\left(Y, \theta_{\phi(e)}, \preceq_{2}\right)$ is I (resp. D, B) -continuous.

Proof. Let $U$ be an open subset of $\left(Y, \theta_{\phi(e)}, \preceq_{2}\right)$. Then there exists a soft open subset $G_{F}$ of $\left(Y, \theta, F, \preceq_{2}\right)$ such that $G(\phi(e))=U$. Since $g_{\phi}$ is a soft I (resp. soft D, soft B) -continuous map, then $g_{\phi}^{-1}\left(G_{F}\right)$ is a soft I (resp. soft D, soft B) -open set. From Definition (1.7), it follows that a soft subset $g_{\phi}^{-1}\left(G_{F}\right)=\left(g_{\phi}^{-1}(G)\right)_{E}$ of $\left(X, \tau, E, \preceq_{1}\right)$ is given by $g_{\phi}^{-1}(G)(e)=g^{-1}(G(\phi(e)))$ for each $e \in E$. This implies a subset $g^{-1}(G(\phi(e)))=g^{-1}(U)$ of $\left(X, \tau_{e}, \preceq_{1}\right)$ is I (resp. D, B) -open. Hence a map $g$ is I (resp. D, B) -continuous.

Theorem 2.5. Let $\tau^{\star}$ be an extended soft topology on $X$. Then a soft map $g_{\phi}:\left(X, \tau^{\star}, E, \preceq_{1}\right) \rightarrow\left(Y, \theta, F, \preceq_{2}\right)$ is soft $I$ (resp. soft $D$, soft B) -continuous if and only if a map $g:\left(X, \tau_{e}^{\star}, \preceq_{1}\right) \rightarrow\left(Y, \theta_{\phi(e)}, \preceq_{2}\right)$ is I (resp. D, B) -continuous.

Proof. The proof of the necessary condition is given above.

Sufficiency: Let $G_{F}$ be a soft open subset of $\left(Y, \theta, F, \preceq_{2}\right)$. Then from Definition (1.7), it follows that a soft subset $g_{\phi}^{-1}\left(G_{F}\right)=\left(g_{\phi}^{-1}(G)\right)_{E}$ of $\left(X, \tau^{\star}, E, \preceq_{1}\right)$ is given by $g_{\phi}^{-1}(G)(e)=g^{-1}(G(\phi(e)))$ for each $e \in E$. Since a map $g$ is I (resp. D, B) -continuous, then a subset $g^{-1}(G(\phi(e)))$ of $\left(X, \tau_{e}^{\star}, \preceq_{1}\right)$ is I (resp. D, B) -open. By hypothesis, $\tau^{\star}$ is an extended soft topology on $X, g_{\phi}^{-1}\left(G_{F}\right)$ is a soft open subset of $\left(X, \tau^{\star}, E, \preceq_{1}\right)$. Hence a soft map $g_{\phi}$ is soft I (resp. soft D, soft B) -continuous.

Theorem 2.6. Let an injective soft map $g_{\phi}:\left(X, \tau, E, \preceq_{1}\right) \rightarrow\left(Y, \theta, F, \preceq_{2}\right)$ be soft B-continuous. If $\left(Y, \theta, F, \preceq_{2}\right)$ is a p-soft $T_{i}$-space, then $\left(X, \tau, E, \preceq_{1}\right)$ is p-soft $T_{i}$-ordered for $i=0,1,2$.

Proof. We prove the theorem in the case of $i=2$ and the others follow similar lines.

For $a \npreceq_{1} b$ in $X$, there exist $x, y \in Y$ such that $g(a)=x, g(b)=y$ and $x \neq y$. By hypothesis, $\left(Y, \theta, F, \preceq_{2}\right)$ is p-soft $T_{2}$, there exist disjoint soft open sets $G_{F}$ and $H_{F}$ containing $x$ and $y$, respectively. Since $g_{\phi}$ is soft $B$-continuous, then $g_{\phi}^{-1}\left(G_{F}\right)$ is soft $I$-open and $g_{\phi}^{-1}\left(H_{F}\right)$ is soft $D$-open subsets of $\widetilde{X}$. Obviously, $g_{\phi}^{-1}\left(G_{F}\right) \widetilde{\bigcap} g_{\phi}^{-1}\left(H_{F}\right)=\widetilde{\emptyset}$. It follows from Proposition (4), that $g_{\phi}^{-1}\left(G_{F}\right)$ and $g_{\phi}^{-1}\left(H_{F}\right)$ containing $a$ and $b$, respectively. Hence $\left(X, \tau, E, \preceq_{1}\right)$ is p-soft $T_{2}$-ordered as required.

Corollary 1. Let an injective soft map $g_{\phi}:\left(X, \tau, E, \preceq_{1}\right) \rightarrow\left(Y, \theta, F, \preceq_{2}\right)$ be soft I-continuous (resp. soft Dcontinuous). If $\left(Y, \theta, F, \preceq_{2}\right)$ is a lower (resp. an upper) p-soft $T_{1}$-space, then $\left(X, \tau, E, \preceq_{1}\right)$ is lower (resp. upper) p-soft $T_{1}$-ordered.

Proposition 6. If a bijective soft map $f_{\phi}:\left(X, \tau, E \preceq_{1}\right) \rightarrow\left(Y, \theta, F, \preceq_{2}\right)$ is soft B-continuous such that $\theta$ is not the indiscrete soft topology, then $\preceq_{1}$ is not linearly ordered.

\section{Soft $\lambda$-open and soft $\lambda$-closed maps}

We begin this section by formulating the concepts of soft $\lambda$-open and soft $\lambda$-closed maps. Then we supply two examples to show the relationships among them. Finally, we give the equivalent conditions for each one of these soft maps and study the image of some soft separation axioms under these soft maps.

Definition 3.1. A soft map $f_{\phi}:\left(X, \tau, E, \preceq_{1}\right) \rightarrow\left(Y, \tau, F, \preceq_{2}\right)$ is called:

(i) Soft I (resp. Soft D, Soft B) -open if the image of every soft open subset of $\tilde{X}$ is a soft I (resp. soft D, soft B) -open subset of $\widetilde{Y}$.

(ii) Soft I (resp. Soft D, Soft B) -closed if the image of every soft closed subset of $\widetilde{X}$ is a soft I (resp. soft D, soft B) -closed subset of $\tilde{Y}$. 
Remark 3.1. From Definition (3.1), we can note the following:

(i) Every soft I (soft D, soft B) -open map is soft open.

(ii) Every soft I (soft D, soft B) -closed map is soft closed.

(iii) Every soft B-open map is soft I-open (soft D-open).

(iv) Every soft B-closed map is soft I-closed (soft D-closed).

In the following two examples, we show that the converse of the three statements in remark above fails.

Example 3.1. Let the two universe sets $X=\{h, i, j\}, Y=\{u, v, w, z\}$ and the two parameters sets $A=\left\{a_{1}, a_{2}\right\}$, $B=\left\{b_{1}, b_{2}\right\}$. Consider a map $\phi: A \rightarrow B$ is defined as, $\phi\left(a_{m}\right)=b_{m}$ for $m \in\{1,2\}$ and a map $f: X \rightarrow Y$ is defined as, $f(h)=w$ and $f(i)=f(j)=z$. We define a partial order relation on $Y$ as $\preceq=\triangle \bigcup\{(v, w),(z, u)\}$ and we define two soft topologies $\tau$ and $\theta$ on $X$ and $Y$, respectively, as $\tau=\left\{\widetilde{\emptyset}, \widetilde{X}, F_{A}\right\}$ and $\theta=\left\{\widetilde{\emptyset}, \widetilde{Y}, H_{B}, K_{B}, L_{B}, O_{B}\right\}$, where $F_{A}=$ $\left\{\left(a_{1},\{h, i\}\right),\left(a_{2},\{i\}\right)\right\}, H_{B}=\left\{\left(b_{1},\{w, z\}\right),\left(b_{2},\{z\}\right)\right\}, K_{B}=\left\{\left(b_{1},\{u, v, w\}\right),\left(b_{2},\{u, v\}\right)\right\}, L_{B}=\left\{\left(b_{1},\{w\}\right),\left(b_{2}, \emptyset\right)\right\}$ and $O_{B}=\left\{\left(b_{1}, Y\right),\left(b_{2},\{u, v, z\}\right)\right\}$. Then one can readily check that a soft map $f_{\phi}: S\left(X_{A}\right) \rightarrow S\left(Y_{B}\right)$ is soft open and soft closed. On the other hand, $f_{\phi}\left(F_{A}\right)=H_{B}$ is neither a soft D-open nor a soft I-open set. Hence $f_{\phi}$ is not soft $I$ (soft D, soft B) -open. Also, $f_{\phi}\left(F_{A}^{c}\right)=K_{B}^{c}$ is neither a soft D-open nor a soft I-closed set. Hence $f_{\phi}$ is not soft $I$ (soft D, soft B) -closed.

Example 3.2. In Example above, if we replace only the partial order relation by $\preceq=\triangle \bigcup\{(v, w)\}($ resp. $\preceq=$ $\triangle \bigcup\{(z, u)\})$, then the soft map $f_{\phi}$ is soft I-open and soft I-closed (resp. soft D-open and soft D-closed), but is not soft B-open and soft B-closed.

Theorem 3.1. The following three properties of a soft map $f_{\phi}:\left(X, \tau, E \preceq_{1}\right) \rightarrow\left(Y, \theta, F, \preceq_{2}\right)$ are equivalent:

(i) $f_{\phi}$ is soft I-open;

(ii) $\operatorname{int}\left(f_{\phi}^{-1}\left(M_{F}\right)\right) \widetilde{\subseteq} f_{\phi}^{-1}\left(M_{F}^{i o}\right)$ for every $M_{F} \widetilde{\subseteq} \widetilde{Y}$;

(iii) $f_{\phi}\left(\operatorname{int}\left(N_{E}\right)\right) \widetilde{\subseteq}\left(f_{\phi}\left(N_{E}\right)\right)^{i o}$ for every $N_{E} \widetilde{\simeq} \widetilde{X}$.

Proof. (i) $\Rightarrow$ (ii): Given a soft subset $M_{F}$ of $\tilde{Y}$, it is obvious that $\operatorname{int}\left(f_{\phi}^{-1}\left(M_{F}\right)\right)$ is a soft open subset of $\widetilde{X}$. Then, by hypothesis, it follows that $f_{\phi}\left(\operatorname{int}\left(f_{\phi}^{-1}\left(M_{F}\right)\right)\right)$ is a soft $I$-open subset of $\widetilde{Y}$. Since $f_{\phi}\left(\operatorname{int}\left(f_{\phi}^{-1}\left(M_{F}\right)\right)\right) \widetilde{\subseteq} f_{\phi}\left(f_{\phi}^{-1}\left(M_{F}\right)\right) \widetilde{\subseteq} M_{F}$, then $\operatorname{int}\left(f_{\phi}^{-1}\left(M_{F}\right)\right) \widetilde{\subseteq} f_{\phi}^{-1}\left(M_{F}^{i o}\right)$.

(ii) $\Rightarrow$ (iii): Given a soft subset $N_{E}$ of $\tilde{X}$, from (ii), we obtain $\operatorname{int}\left(f_{\phi}^{-1}\left(f_{\phi}\left(N_{E}\right)\right)\right) \widetilde{\widetilde{C}} f_{\phi}^{-1}\left(\left(f_{\phi}\left(N_{E}\right)\right)^{i o}\right)$. Since $\operatorname{int}\left(N_{E}\right) \widetilde{\subseteq} f_{\phi}^{-1}\left(f_{\phi}\left(\operatorname{int}\left(f_{\phi}^{-1}\left(f_{\phi}\left(N_{E}\right)\right)\right)\right)\right) \widetilde{\subseteq} f_{\phi}^{-1}\left(\left(f_{\phi}\left(N_{E}\right)\right)^{i o}\right)$, then $f_{\phi}\left(\operatorname{int}\left(N_{E}\right)\right) \widetilde{\subseteq}\left(f_{\phi}\left(N_{E}\right)\right)^{i o}$ as required.

(iii) $\Rightarrow$ (i): Let $G_{E}$ be a soft open subset of $\widetilde{X}$. Then $f_{\phi}\left(\operatorname{int}\left(G_{E}\right)\right)=f_{\phi}\left(G_{E}\right) \widetilde{\widetilde{\subseteq}}\left(f_{\phi}\left(G_{E}\right)\right)^{i o}$. Hence $f_{\phi}$ is a soft $I$-open map.

The following theorem can be proved in a similar manner.

Theorem 3.2. The following three properties of a soft map $f_{\phi}:\left(X, \tau, E \preceq_{1}\right) \rightarrow\left(Y, \theta, F, \preceq_{2}\right)$ are equivalent:

(i) $f_{\phi}$ is soft D-open (resp. soft B-open);

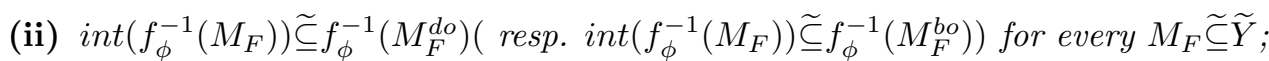

(iii) $f_{\phi}\left(\operatorname{int}\left(N_{E}\right)\right) \widetilde{\subseteq}\left(f_{\phi}\left(N_{E}\right)\right)^{d o}\left(\operatorname{resp} . f_{\phi}\left(\operatorname{int}\left(N_{E}\right)\right) \widetilde{\subseteq}\left(f_{\phi}\left(N_{E}\right)\right)^{b o}\right)$ for every $N_{E} \widetilde{\simeq} \widetilde{X}$.

Theorem 3.3. The following three statements hold for a soft map $f_{\phi}:\left(X, \tau, E \preceq_{1}\right) \rightarrow\left(Y, \theta, F, \preceq_{2}\right)$ :

(i) $f_{\phi}$ is soft I-closed if and only if $\left(f_{\phi}\left(G_{E}\right)\right)^{i c l} \widetilde{\subseteq} f_{\phi}\left(c l\left(G_{E}\right)\right)$ for every $G_{E} \widetilde{\subseteq} \widetilde{X}$.

(ii) $f_{\phi}$ is soft $D$-closed if and only if $\left(f_{\phi}\left(G_{E}\right)\right)^{d c l} \widetilde{\subseteq} f_{\phi}\left(c l\left(G_{E}\right)\right)$ for every $G_{E} \widetilde{\subseteq} \widetilde{X}$.

(iii) $f_{\phi}$ is soft $B$-closed if and only if $\left(f_{\phi}\left(G_{E}\right)\right)^{b c l} \widetilde{\subseteq} f_{\phi}\left(c l\left(G_{E}\right)\right)$ for every $G_{E} \widetilde{\subseteq} \widetilde{X}$. 
Proof. We only give a proof for the first statement and the others follow similar lines.

Necessity: Since $f_{\phi}$ is soft $I$-closed, then $f_{\phi}\left(\operatorname{cl}\left(G_{E}\right)\right)$ is a soft $I$-closed subset of $\widetilde{Y}$ and since $f_{\phi}\left(G_{E}\right) \widetilde{\subseteq} f_{\phi}\left(c l\left(G_{E}\right)\right)$, then $\left(f_{\phi}\left(G_{E}\right)\right)^{i c l} \widetilde{\simeq} f_{\phi}\left(c l\left(G_{E}\right)\right)$.

Sufficiency: Consider $H_{E}$ is a soft closed subset of $\widetilde{X}$. Then $f_{\phi}\left(H_{E}\right) \widetilde{\subseteq}\left(f_{\phi}\left(H_{E}\right)\right)^{i c l} \widetilde{\subseteq} f_{\phi}\left(c l\left(H_{E}\right)\right)=f_{\phi}\left(H_{E}\right)$. Therefore $f_{\phi}\left(H_{E}\right)=\left(f_{\phi}\left(H_{E}\right)\right)^{i c l}$. This means that $f_{\phi}\left(H_{E}\right)$ is a soft $I$-closed set. Hence the proof is complete.

Theorem 3.4. The following three statements hold for a bijective soft map $f_{\phi}:\left(X, \tau, E \preceq_{1}\right) \rightarrow\left(Y, \theta, F, \preceq_{2}\right)$ :

(i) $f_{\phi}$ is soft I (resp. soft D, soft B) -open if and only if $f_{\phi}$ is soft D (resp. soft D, soft B) -closed.

(ii) $f_{\phi}$ is soft I (resp. soft D, soft B) -open if and only if $f_{\phi}^{-1}$ is soft I (resp. soft D, soft B) -continuous.

(iii) $f_{\phi}$ is soft $D$ (resp. soft I, soft B) -closed if and only if $f_{\phi}^{-1}$ is soft I (resp. soft D, soft B) -continuous.

Proof. For the sake of economy, we only give proofs of cases outside the parenthesis for the three statements in and the cases between parenthesis can be made similarly.

(i) To prove the necessary condition, let $H_{E}$ be a soft closed subset of $\widetilde{X}$ and consider $f_{\phi}$ is a soft $I$-open map. Then $H_{E}^{c}$ is soft open and $f_{\phi}\left(H_{E}^{c}\right)$ is soft $I$-open. It follows from the bijectiveness of $f_{\phi}$, that $f_{\phi}\left(H_{E}^{c}\right)=\left[f_{\phi}\left(H_{E}\right)\right]^{c}$. This automatically implies that $f_{\phi}\left(H_{E}\right)$ is soft $D$-closed. Thus $f_{\phi}$ is a soft $D$-closed map. In a similar manner, we can prove the sufficiency condition.

(ii) Necessity: Let $G_{E}$ be a soft open subset of $\widetilde{X}$ and consider $f_{\phi}$ is a soft $I$-open map. Then $f_{\phi}\left(G_{E}\right)$ is soft $I$-open. It follows from the bijectiveness of $f_{\phi}$, that $f_{\phi}\left(G_{E}\right)=\left(f_{\phi}^{-1}\right)^{-1}\left(G_{E}\right)$. This automatically implies that $\left(f_{\phi}^{-1}\right)^{-1}\left(G_{E}\right)$ is soft $I$-open. Thus $f_{\phi}^{-1}$ is a soft $I$-continuous map. In a similar manner, we can prove the sufficiency condition.

(iii) The proof of this statement comes immediately from (i) and (ii) above.

Theorem 3.5. If a soft map $g_{\phi}:\left(X, \tau, E, \preceq_{1}\right) \rightarrow\left(Y, \theta, F, \preceq_{2}\right)$ is soft $I$ (resp. soft D, soft B) -open and $\phi$ is an injective map, then a map $g:\left(X, \tau_{e}, \preceq_{1}\right) \rightarrow\left(Y, \theta_{\phi(e)}, \preceq_{2}\right)$ is $I$ (resp. D, B) -open.

Proof. Let $U$ be an open subset of $\left(X, \tau_{e}, \preceq_{1}\right)$ and $\phi(e)=f$. Then there exists a soft open subset $G_{E}$ of $\left(X, \tau, E, \preceq_{1}\right)$ such that $G(e)=U$. Since $g_{\phi}$ is a soft I (resp. soft D, soft B) -open map, then $g_{\phi}\left(G_{E}\right)$ is a soft I (resp. soft D, soft B) -open set. From Definition (1.7), it follows that a soft subset $g_{\phi}\left(G_{E}\right)=\left(g_{\phi}(G)\right)_{F}$ of $\left(Y, \theta, F, \preceq_{2}\right)$ is given by $g_{\phi}(G)(f)=\bigcup_{e \in \phi^{-1}(f)} g(G(e))$ for each $f \in F$. This implies a subset $\bigcup_{e \in \phi^{-1}(f)} g(G(e))=g(U)$ of $\left(Y, \theta_{\phi(e)}, \preceq_{2}\right)$ is I (resp. D, B) -open. Hence a map $g$ is I (resp. D, B) -open.

Theorem 3.6. Let $\theta^{\star}$ be an extended soft topology on $Y$ and $\phi$ be an injective map. Then a soft map $g_{\phi}:(X, \tau, E, \preceq 1$ )$\rightarrow\left(Y, \theta^{\star}, F, \preceq_{2}\right)$ is soft I (resp. soft $D$, soft B) -open if and only if a map $g:\left(X, \tau_{e}, \preceq_{1}\right) \rightarrow\left(Y, \theta_{\phi}^{\star}(e), \preceq_{2}\right)$ is $I$ (resp. $D, B)$-open.

Proof. The proof of the necessary condition is given above.

Sufficiency: Let $G_{E}$ be a soft open subset of $\left(X, \tau, E, \preceq_{1}\right)$. Then from Definition (1.7), it follows that a soft subset $g_{\phi}\left(G_{E}\right)=\left(g_{\phi}(G)\right)_{F}$ of $\left(Y, \theta^{\star}, F, \preceq_{2}\right)$ is given by $g_{\phi}(G)(f)=\bigcup_{e \in \phi^{-1}(f)} g(G(e))$ for each $f \in F$. Since a map $g$ is I (resp. D, B) -open, then a subset $\bigcup_{e \in \phi^{-1}(f)} g(G(e))$ of $\left(Y, \theta_{\phi(e)}^{\star}, \preceq_{2}\right)$ is I (resp. D, B) -open. By hypothesis, $\theta^{\star}$ is an extended soft topology on $Y, g_{\phi}\left(G_{E}\right)$ is a soft subset of $\left(Y, \theta^{\star}, F, \preceq_{2}\right)$. Hence a soft map $g_{\phi}$ is soft I (resp. soft D, soft B) -open.

The two theorems above are restated in the case of a soft I (resp. soft D, soft B) -closed map. One can prove them similarly and so the proof will be omitted.

Theorem 3.7. If a soft map $g_{\phi}:\left(X, \tau, E, \preceq_{1}\right) \rightarrow\left(Y, \theta, F, \preceq_{2}\right)$ is soft I (resp. soft D, soft B) -closed and $\phi$ is an injective map, then a map $g:\left(X, \tau_{e}, \preceq_{1}\right) \rightarrow\left(Y, \theta_{\phi(e)}, \preceq_{2}\right)$ is $I$ (resp. D, B) -closed.

Theorem 3.8. Let $\theta^{\star}$ be an extended soft topology on $Y$ and $\phi$ is an injective map. Then a soft map $g_{\phi}:\left(X, \tau, E, \preceq_{1}\right.$ )$\rightarrow\left(Y, \theta^{\star}, F, \preceq_{2}\right)$ is soft I (resp. soft $D$, soft B) -closed if and only if a map $g:\left(X, \tau_{e}, \preceq_{1}\right) \rightarrow\left(Y, \theta_{\phi(e)}^{\star}, \preceq_{2}\right)$ is I (resp. $D, B)$-closed. 
Theorem 3.9. Let a bijective soft map $g_{\phi}:\left(X, \tau, E, \preceq_{1}\right) \rightarrow\left(Y, \theta, F, \preceq_{2}\right)$ be soft B-open (resp. soft B-closed). If $\left(X, \tau, E, \preceq_{1}\right)$ is a $p$-soft $T_{i}$-space, then $\left(Y, \theta, F, \preceq_{2}\right)$ is $p$-soft $T_{i}$-ordered for $i=0,1,2$.

Proof. We prove the theorem in the case of $i=2$ and the others follow similar lines.

For $x \npreceq_{1} y$ in $Y$, there exist $a, b \in X$ such that $g^{-1}(x)=a, g^{-1}(y)=b$ and $a \neq b$. By hypothesis, $\left(X, \tau, E, \preceq_{1}\right)$ is p-soft $T_{2}$, there exist disjoint soft open sets $G_{F}$ and $H_{F}$ containing $a$ and $b$, respectively. Since $g_{\phi}$ is soft $B$-open, then $g_{\phi}\left(G_{F}\right)$ is soft $I$-open and $g_{\phi}\left(H_{F}\right)$ is soft $D$-open subsets of $\widetilde{Y}$. The bijective of $g_{\phi}$ implies that $g_{\phi}\left(G_{F}\right) \widetilde{\bigcap} g_{\phi}\left(H_{F}\right)=\widetilde{\emptyset}$. It follows from Proposition (4), that $g_{\phi}\left(G_{F}\right)$ and $g_{\phi}\left(H_{F}\right)$ containing $x$ and $y$, respectively. Hence $\left(Y, \theta, F, \preceq_{2}\right)$ is p-soft $T_{2}$-ordered as required.

Corollary 2. Let a bijective soft map $g_{\phi}:\left(X, \tau, E, \preceq_{1}\right) \rightarrow\left(Y, \theta, F, \preceq_{2}\right)$ be soft I-open or soft D-closed (resp. soft $D$-open or soft $I$-closed). If $\left(X, \tau, E, \preceq_{1}\right)$ is a lower (resp. an upper) p-soft $T_{1}$-space, then $\left(Y, \theta, F, \preceq_{2}\right)$ is lower (resp. upper) $p$-soft $T_{1}$-ordered.

Proposition 7. Consider $\tau$ is not the indiscrete soft topology on $X$. If an injective soft map $f_{\phi}:\left(X, \tau, E \preceq{ }_{1}\right) \rightarrow$ $\left(Y, \theta, F, \preceq_{2}\right)$ is soft B-open or soft B-closed, then $\preceq_{2}$ is not linearly ordered.

\section{Soft $\lambda$-homeomorphism maps}

In this section, the concepts of soft $\lambda$-homeomorphism maps are established and their main properties are discussed. The image and pre image of p-soft $T_{i}$ and p-soft $T_{i}$-ordered under the these soft maps are investigated and some compositions soft maps are constructed.

Definition 4.1. A bijective soft map $g_{\phi}:\left(X, \tau, E, \preceq_{1}\right) \rightarrow\left(Y, \theta, F, \preceq_{2}\right)$ is called soft I (resp. soft D, soft B) homeomorphism if it is soft I-continuous and soft I-open (resp. soft D-continuous and soft D-open, soft B-continuous and soft B-open).

Remark 4.1. From Definition 4.1), we can note the following:

(i) Every soft I (soft D, soft B) -homeomorphism map is soft homeomorphism.

(ii) Every soft B-homeomorphism map is soft I-homeomorphism (soft D-homeomorphism).

To elucidate that the two items of the remark above are not conversely, we give two examples below.

Example 4.1. Let the two universe sets $X=\{h, i\}, Y=\{w, z\}$ and the two parameters sets $A=\left\{a_{1}, a_{2}\right\}$, $B=\left\{b_{1}, b_{2}\right\}$. Consider a map $\phi: A \rightarrow B$ is defined as, $\phi\left(a_{m}\right)=b_{m}$ for $m \in\{1,2\}$ and a map $f: X \rightarrow Y$ is defined as, $f(h)=w$ and $f(i)=z$. We define two partial order relations on $X$ and $Y$, respectively, as $\preceq_{1}=\triangle \bigcup\{(h, i)\}$ and $\preceq_{2}=\triangle \bigcup\{(z, w)\}$ and we define two soft topologies $\tau$ and $\theta$ on $X$ and $Y$, respectively, as $\tau=\left\{\widetilde{\emptyset}, \widetilde{X}, F_{A}\right\}$ and $\theta=\left\{\widetilde{\emptyset}, \widetilde{Y}, H_{B}\right\}$, where $F_{A}=\left\{\left(a_{1}, X\right),\left(a_{2},\{h\}\right)\right\}$ and $H_{B}=\left\{\left(b_{1}, Y\right),\left(b_{2},\{w\}\right)\right\}$. Then one can readily check that a soft map $f_{\phi}: S\left(X_{A}\right) \rightarrow S\left(Y_{B}\right)$ is soft homeomorphism. On the other hand, $f_{\phi}\left(F_{A}\right)=H_{B}$ is not a soft D-open set and $f_{\phi}^{-1}\left(H_{B}\right)=F_{A}$ is not a soft I-open set. Hence $f_{\phi}$ is not soft I (soft D, soft B) -homeomorphism.

Example 4.2. In Example above, if we replace only the partial order relation $\varliminf_{2}$ by $\preceq=\triangle \bigcup\{(w, z)\}$, then the soft map $f_{\phi}$ is soft D-homeomorphism, but is not soft B-homeomorphism. Also, if we replace only the partial order relation $\preceq_{1}$ by $\preceq=\triangle \bigcup\{(i, h)\}$, then the soft map $f_{\phi}$ is soft I-homeomorphism, but is not soft B-homeomorphism.

Theorem 4.1. Consider $f_{\phi}:\left(X, \tau, E, \preceq_{1}\right) \rightarrow\left(Y, \theta, F, \preceq_{2}\right)$ is a bijective soft map and let $(\lambda, \gamma) \in\{(I, d c l),(D, i c l),(B, b c l)\}$. Then $f_{\phi}$ is soft $\lambda$-homeomorphism if and only if $\left(f_{\phi}\left(G_{E}\right)\right)^{\gamma}=f_{\phi}\left(\operatorname{cl}\left(G_{E}\right)\right)=\operatorname{cl}\left(f_{\phi}\left(G_{E}\right)\right)=f_{\phi}\left(G_{E}^{\gamma}\right)$ for every $G_{E} \widetilde{\subseteq} \widetilde{X}$.

Proof. We make a proof for the theorem in the case of $(\lambda, \gamma)=(I, d c l)$ and the other follow similar line.

Necessity: The property of $f_{\phi}$ is a soft $I$-homeomorphism map implies that $f_{\phi}\left(G_{E}^{d c l}\right) \widetilde{\subseteq} c l\left(f_{\phi}\left(G_{E}\right)\right)$ and $\left(f_{\phi}\left(G_{E}\right)\right)^{d c l} \widetilde{\subseteq} f_{\phi}\left(c l\left(G_{E}\right)\right)$

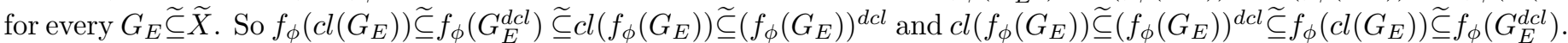
By the preceding two inclusion relations, we obtain the required equality relation.

Sufficiency: The equality relation $\left(f_{\phi}\left(G_{E}\right)\right)^{d c l}=f_{\phi}\left(c l\left(G_{E}\right)\right)=\operatorname{cl}\left(f_{\phi}\left(G_{E}\right)\right)=f_{\phi}\left(G_{E}^{d c l}\right)$ implies that $f_{\phi}\left(G_{E}^{d c l}\right) \widetilde{\widetilde{c}} c l\left(f_{\phi}\left(G_{E}\right)\right)$ and $\left(f_{\phi}\left(G_{E}\right)\right)^{d c l} \widetilde{\simeq} f_{\phi}\left(c l\left(G_{E}\right)\right)$. So $f_{\phi}$ is soft $I$-continuous and soft $D$-closed map. Hence the desired result is proved. 
Theorem 4.2. If a bijective soft map $f_{\phi}:\left(X, \tau, E, \preceq_{1}\right) \rightarrow\left(Y, \theta, F, \preceq_{2}\right)$ is soft $I$-continuous (resp. soft D-continuous, soft $B$-continuous), Then the following three statements are equivalent:

(i) $f_{\phi}$ is soft I-homeomorphism (resp. soft D-homeomorphism, soft B-homeomorphism );

(ii) $f_{\phi}^{-1}$ is soft $I$-continuous (resp. soft D-continuous, soft B-continuous);

(iii) $f_{\phi}$ is soft D-closed (resp. soft I-closed, soft B-closed).

Proof. (i) $\Rightarrow$ (ii) Since $f_{\phi}$ is a soft $I$-homeomorphism (resp. soft $D$-homeomorphism, soft $B$-homeomorphism) map, then $f_{\phi}$ is soft $I$-open (resp. soft $D$-open, soft $B$-open). It follows from item (ii) of Theorem $(3.4)$, that $f_{\phi}^{-1}$ is soft $I$-continuous (resp. soft $D$-continuous, soft $B$-continuous).

(ii) $\Rightarrow$ (iii) The proof follows from item (iii) of Theorem 3.4.

(iii) $\Rightarrow$ (i) It sufficient to prove that $f_{\phi}$ is a soft $I$-open (resp. soft $D$-open, soft $B$-open) map. This follows from item (i) of Theorem (3.4).

Theorem 4.3. If a soft map $g_{\phi}:\left(X, \tau, E, \preceq_{1}\right) \rightarrow\left(Y, \theta, F, \preceq_{2}\right)$ is soft $I$ (resp. soft D, soft B) -homeomorphism, then a map $g:\left(X, \tau_{e}, \preceq_{1}\right) \rightarrow\left(Y, \theta_{\phi(e)}, \preceq_{2}\right)$ is $I$ (resp. D, B) -homeomorphism.

Proof. The proof is obtained immediately from Theorem 2.4 and Theorem 3.5

Theorem 4.4. Let $\tau^{\star}$ and $\theta^{\star}$ be extended soft topologies on $X$ and $Y$, respectively. Then a soft map $g_{\phi}:\left(X, \tau^{\star}, E, \preceq_{1}\right.$ )$\rightarrow\left(Y, \theta^{\star}, F, \preceq_{2}\right)$ is soft I (resp. soft D, soft B) -homeomorphism if and only if a map $g:\left(X, \tau_{e}^{\star}, \preceq_{1}\right) \rightarrow\left(Y, \theta_{\phi}^{\star}(e), \preceq_{2}\right)$ is $I$ (resp. D, B) -homeomorphism.

Proof. The proof is obtained immediately from Theorem 2.5 and Theorem 3.6

Theorem 4.5. Let a soft map $g_{\phi}:\left(X, \tau, E, \preceq_{1}\right) \rightarrow\left(Y, \theta, F, \preceq_{2}\right)$ be soft B-homeomorphism. Then $\left(X, \tau, E, \preceq_{1}\right)$ is a $p$-soft $T_{i}$-space if and only if $\left(Y, \theta, F, \preceq_{2}\right)$ is $p$-soft $T_{i}$-ordered for $i=0,1,2,3,4$.

Proof. The proof of the theorem in the cases of $i=0,1,2$ follows from Theorem (2.6) and Theorem (3.9).

To prove the theorem in the case $i=3$, let $F_{E}$ be a soft closed subset of $\widetilde{X}$ such that $a \notin F_{E}$. Then $g_{\phi}\left(F_{E}\right)$ is a soft balancing closed subset of $\tilde{Y}$ such that $g(a) \notin g_{\phi}\left(F_{E}\right)$. By hypothesis, $\left(Y, \theta, F, \preceq_{2}\right)$ is p-soft regular ordered, there exist disjoint soft open sets $G_{F}$ and $H_{F}$ containing $g(a)$ and $g_{\phi}\left(F_{E}\right)$, respectively. So $g_{\phi}^{-1}\left(G_{F}\right)$ and $g_{\phi}^{-1}\left(H_{F}\right)$ are disjoint soft open sets containing $a$ and $F_{E}$, respectively. Thus the necessary condition holds. Conversely, let $L_{F}$ be an increasing or decreasing soft closed subset of $\widetilde{Y}$ such that $x \notin L_{F}$. Say, $L_{F}$ is increasing soft closed. Then $g_{\phi}^{-1}\left(L_{F}\right)$ is a soft closed set and $g^{-1}(x) \notin g_{\phi}^{-1}\left(L_{F}\right)$. By hypothesis, $\left(X, \tau, E, \preceq_{1}\right)$ is p-soft regular, there exist disjoint soft open sets $G_{E}$ and $H_{E}$ containing $g^{-1}(x)$ and $g_{\phi}^{-1}\left(L_{F}\right)$, respectively. So $g_{\phi}\left(G_{F}\right)$ and $g_{\phi}\left(H_{F}\right)$ are disjoint soft open sets containing $x$ and $L_{F}$, respectively, such that $g_{\phi}\left(G_{F}\right)$ is decreasing and $g_{\phi}\left(H_{F}\right)$ is increasing. Hence the desired result is proved.

One can made similarly the theorem's proof for $i=4$.

Corollary 3. Let a soft map $g_{\phi}:\left(X, \tau, E, \preceq_{1}\right) \rightarrow\left(Y, \theta, F, \preceq_{2}\right)$ be soft I-homeomorphism (resp. soft D-homeomorphism). Then $\left(X, \tau, E, \preceq_{1}\right)$ is a lower (resp. an upper) p-soft $T_{1}$-space if and only if $\left(Y, \theta, F, \preceq_{2}\right)$ is lower (resp. upper) p-soft $T_{1}$-ordered.

Corollary 4. Let a soft map $g_{\phi}:\left(X, \tau, E, \preceq_{1}\right) \rightarrow\left(Y, \theta, F, \preceq_{2}\right)$ be soft I-homeomorphism (resp. soft D-homeomorphism). Then $\left(X, \tau, E, \preceq_{1}\right)$ is an upper (resp. a lower) p-soft regular if and only if $\left(Y, \theta, F, \preceq_{2}\right)$ is an upper (resp. a lower) p-soft regular ordered.

Definition 4.2. The composition of two soft maps $g_{\lambda}:\left(X, \tau, E, \preceq_{1}\right) \rightarrow\left(Y, \theta, F, \preceq_{2}\right)$ and $f_{\phi}:\left(Y, \theta, F, \preceq_{2}\right) \rightarrow$ $\left(Z, v, K, \preceq_{3}\right)$ is a soft map $f_{\phi} \circ g_{\lambda}:\left(X, \tau, E, \preceq_{1}\right) \rightarrow\left(Z, v, K, \preceq_{3}\right)$ and is given by $\left(f_{\phi} \circ g_{\lambda}\right)\left(P_{e}^{x}\right)=f_{\phi}\left(g_{\lambda}\left(P_{e}^{x}\right)\right)$.

Proposition 8. Let $f_{\phi}:\left(X, \tau, E, \preceq_{1}\right) \rightarrow\left(Y, \theta, F, \preceq_{2}\right)$ and $g_{\eta}:\left(Y, \theta, F, \preceq_{2}\right) \rightarrow\left(Z, v, K, \preceq_{3}\right)$ be two soft maps. Then then following properties hold for $\lambda \in\{I, D, B\}$.

(i) If $g_{\eta}$ is a soft $\lambda$-homeomorphism map and $f_{\phi}$ is an increasing soft homeomorphism map, then $g_{\eta} \circ f_{\phi}$ is a soft $\lambda$-homeomorphism map.

(ii) If $f_{\phi}$ is a soft $\lambda$-continuous map and $g_{\eta}$ is a soft continuous map, then $g_{\eta} \circ f_{\phi}$ is a soft $\lambda$-continuous map. 
(iii) If $f_{\phi}$ is a soft open (resp. soft closed) map and $g_{\eta}$ is a soft $\lambda$-open (resp. soft $\lambda$-open) map, then $g_{\eta} \circ f_{\phi}$ is a soft $\lambda$-open (resp. soft $\lambda$-closed) map.

(iv) If $g_{\eta} \circ f_{\phi}$ is a soft $\lambda$-open map and $f_{\phi}$ is surjective soft continuous, then $g_{\eta}$ is a soft $\lambda$-open map.

(v) If $g_{\eta} \circ f_{\phi}$ is a soft closed map and $g_{\eta}$ is an injective soft $\lambda$-continuous map, then $f_{\phi}$ is a soft $\gamma$-closed map, where $(\lambda, \gamma) \in\{(I, D),(D, I),(B, B)\}$.

Proposition 9. Let $\tau$ and $\theta$ be two soft topologies on $X$ and $Y$, respectively, such that they do not belong to $\{$ discrete soft topology, indiscrete soft topology\}. If a soft map $f_{\phi}:\left(X, \tau, E \preceq_{1}\right) \rightarrow\left(Y, \theta, F, \preceq_{2}\right)$ is soft B-homeomorphism, then $\preceq_{1}$ and $\preceq_{2}$ are not linearly ordered.

\section{Conclusion}

Al-shami et al. 9] initiated the notion of a soft topological ordered space by equipping a soft partially ordered set with a soft topological space. As a continuation of this, we present in this manuscript, the concepts of soft $\lambda$-continuous, soft $\lambda$-open, soft $\lambda$-closed and soft $\lambda$-homeomorphism maps, where $\lambda \in\{I, D, B\}$. To obtain a deeper understanding of these concepts, we give some examples which show the relationships among them and illustrate that they are strictly stronger than their counterparts on topological ordered spaces. Also, we characterize each one of the introduced soft maps and deduce some results which connect them with those maps on topological ordered spaces. Moreover, we probe the behavior of p-soft $T_{i}$ and p-soft $T_{i}$-ordered spaces under these soft maps and study some compositions of the introduced soft maps. In the end, we hope that the concepts presented herein give rise to constitute fundamental background for studying several topics on soft topological ordered spaces.

ACKNOWLEDGEMENTS. The authors thank the referees for their comments and suggestions that improved this article. The authors thank the ambassador of Republic of Yemen in Egypt Dr. Mohammed Ali Maram for his continuous encouragement and support.

\section{References}

[1] M. Abbas, B. Ali and S. Romaguera, On generalized soft equality and soft lattice structure, Filomat, 28 (6) (2014) 1191-1203.https://doi.org/10.2298/fil1406191a

[2] M. Abbas, M. I. Ali and S. Romaguera, Generalized operations in soft set theory via relaxed conditions on parameters, Filomat, 31(19) (2017) 5955-5964.https://doi.org/10.2298/fil1719955a

[3] M. Abo-Elhamayel and T. M. Al-shami, Supra homeomorphism in supra topological ordered spaces, Facta Universitatis, Series: Mathematics and Informatics, 31 (5) (2016) 10911106.https://doi.org/10.22190/fumi1605091a

[4] M. I. Ali, F. Feng, X. Liu, W. K. Min and M. Shabir, On some new operations in soft set theory, Computers and Mathematics with Applications, 57 (2009) 1547-1553.https://doi.org/10.1016/j.camwa.2008.11.009

[5] T. M. Al-shami, Supra $\beta$-bicontinuous maps via topological ordered spaces, Mathematical Sciences Letters, 6 (3) (2017) 239-247.https://doi.org/10.18576/msl/060304

[6] T. M. Al-shami, Soft somewhere dense sets on soft topological spaces, Communications of the Korean Mathematical Society, 33 (4) (2018) 1341-1356.

[7] T. M. Al-shami, On some maps in supra topological ordered spaces, Journal of New Theory, 20 (2018) 76-92.

[8] T. M. Al-shami and M. K. Tahat, I (D, B)-supra pre maps via supra topological ordered spaces, Journal of Progressive Research in Mathematics, 12 (3) (2017) 1989-2001.

[9] T. M. Al-shami, M. E. El-Shafei and M. Abo-Elhamayel, On soft topological ordered spaces, Journal of King Saud University-Science, https://doi.org/10.1016/j.jksus.2018.06.005. 
[10] T. M. Al-shami, M. E. El-Shafei and M. Abo-Elhamayel, Almost soft compact and approximately soft Lindelöf spaces, Journal of Taibah University for Science, 12 (5) (2018) 620630.https://doi.org/10.1080/16583655.2018.1513701

[11] S. D. Arya and K. Gupta, New separation axioms in topological ordered spaces, Indain Journal Pure and Appllied Mathematics, 22 (1991) 461-468.

[12] A. Aygünoğlu and H. Aygün, Some notes on soft topological spaces, Neural Computers and Applications, 21 (2012) 113-119.https://doi.org/10.1007/s00521-011-0722-3

[13] K. V. Babitha and J.J. Sunil, Soft set relations and functions, Computers and Mathematics with Applications, 60 (2010) 1840-1849.https://doi.org/10.1016/j.camwa.2010.07.014

[14] P. Das, Separation axioms in ordered spaces, Soochow Journal of Mathematics, 30 (4) (2004) 447-454.

[15] S. Das and S. K. Samanta, Soft metric, Annals of Fuzzy Mathematics and Informatics, 1 (2013) 77-94.

[16] M. E. El-Shafei, M. Abo-Elhamayel and T. M. Al-shami, Strong separation axioms in supra topological ordered spaces, Mathematical Sciences Letters, 6 (3) (2017) 271-277.https://doi.org/10.18576/msl/060308

[17] M. E. El-Shafei, M. Abo-Elhamayel and T. M. Al-shami, Supra R-homeomorphism in supra topological ordered spaces, International Journal of Algebra and Statistics, 6 (1-2) (2017) 158167.https://doi.org/10.20454/ijas.2017.1331

[18] M. E. El-Shafei, M. Abo-Elhamayel and T. M. Al-shami, Generating ordered maps via supra topological ordered spaces, International Journal of Modern Mathematical Sciences, 15 (3) (2017) 339-357.

[19] M. E. El-Shafei, M. Abo-Elhamayel and T. M. Al-shami, Partial soft separation axioms and soft compact spaces, Filomat, 32 (13) (2018) 4755-4771.

[20] F. Feng and Y. M. Li, Soft subsets and soft product operations, Information Science, 232 (2013) 14681470.https://doi.org/10.1016/j.ins.2013.01.001

[21] F. Feng, Y. M. Li, B. Davvaz and M. I. Ali, Soft sets combined with fuzzy sets and rough sets: a tentative approach, Soft Comput, 14 (2010) 899-911.https://doi.org/10.1007/s00500-009-0465-6

[22] M. H. Ghanim, On some types of topologies, Master Thesis, Al-Azher University, (1976).

[23] T. Hida, A comprasion of two formulations of soft compactness, Annals of Fuzzy Mathematics and Informatics, $8(4)(2014) 511-524$.

[24] M. K. R. S. V. Kumar, Homeomorphism in topological ordered spaces, Acta Ciencia Indian, XXVIII(M)(1)(2012) 67-76.

[25] H. P. A. Künzi, Completely regular ordered spaces, Order, 7 (1990) 283-293.https://doi.org/10.1007/bf00418656

[26] H. P. A. Künzi and T. A. Richmond, Completely regularly ordered spaces versus $T_{2}$-ordered spaces which are completely regular, Topology and its Applications, 135 (2004) 185-196.https://doi.org/10.1016/s01668641(03)00162-7

[27] D. S. Leela and G. Balasubramanian, New separation axioms in ordered topological spaces, Indian Journal of Pure and Applied Mathematics, 33 (2002) 1011-1016.

[28] F. Li, Notes on the soft operations, ARPN Journal of Systems and Software, 1 (6) (2011) 205-208.

[29] X. Liu and F. Feng, Y. B. Jun, A note on generalized soft equal relations, Computers and Mathematics with Applications, 64 (2012) 572-578.https://doi.org/10.1016/j.camwa.2011.12.052

[30] P. K. Maji, R. Biswas and R. Roy, Soft set theory, Computers and Mathematics with Applications, 45 (2003) 555-562. 
[31] S. D. McCartan, Separation axioms for topological ordered spaces, Mathematical Proceedings of the Cambridge Philosophical Society, 64 (1986) 965-973.https://doi.org/10.1017/s0305004100043668

[32] D. Molodtsov, Soft set theory-first results, Computers and Mathematics with Applications, 37 (1999) 1931.https://doi.org/10.1016/s0898-1221(99)00056-5

[33] L. Nachbin, Topology and ordered, D. Van Nostrand Inc. Princeton, New Jersey, (1965).

[34] S. Nazmul and S. K. Samanta, Neigbourhood properties of soft topological spaces, Annals of Fuzzy Mathematics and Informatics, 6 (1) (2013) 1-15.

[35] T. Y. Ozturk and S. Bayramov, Soft mappings space, The Scientific World Journal, Volume 2014, Article ID 307292, 8 pages.https://doi.org/10.1155/2014/307292

[36] D. Pei and D. Miao, From soft sets to information system, In Proceedings of the IEEE International Conference on Granular Computing, 2 (2005) 617-621.https://doi.org/10.1109/grc.2005.1547365

[37] M. Shabir and M. Naz, On soft topological spaces, Computers and Mathematics with Applications, 61 (2011) 1786-1799.

[38] I. Zorlutuna, M. Akdag, W. K. Min and S. K. Samanta, Remarks on soft topological spaces, Annals of Fuzzy Mathematics and Informatics, 2 (2012) 171-185. 